\title{
Sepsis, one of CMAJ's four new areas of focus
}

\author{
Diane Kelsall MD MEd, Niranjan Kissoon MBBS
}

- Cite as: CMAJ 2017 September 11;189:E1127. doi: 10.1503/cmaj.171006

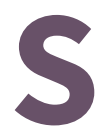

ometimes readers and authors ask what CMAJ is especially interested in. Which trends in health care concern us? What emerging issues do we worry about? As a general medical journal, CMAJ covers a wide range of medical topics for a broad readership in Canada and internationally. And with our far-reaching vision to champion knowledge that matters for the health of Canadians and the rest of the world, perhaps it can be difficult to tell what keeps us up at night.

The CMAJ editorial team and the Editorial Advisory Board have honed in on four areas of focus: mental health, health of vulnerable populations, sepsis, and health services and quality of care. In this issue, we launch our focus on sepsis, the final common pathway to death from infection. ${ }^{1}$

A 1911 CMAJ article describing the typical clinical course of suppurative nephritis concluded thus: "the child usually dies in extreme exhaustion within a few days." ${ }^{2}$ It's a chilling report of sepsis in an age with very limited treatment options. But the concept of sepsis dates back to Hippocrates' first use of the term for a fearsome complication of wounds leading to death. Prior to the recognition of causative infectious agents and antimicrobial therapy, sepsis played a decisive role in geographic colonization and the outcomes of wars.

Sepsis is now defined as a dysregulated immune response to infections, leading to organ failure and death. ${ }^{3}$ An estimated 8 million people die each year from sepsis, mainly from community-acquired infection. Most of these deaths are preventable., ${ }^{1,4}$

Describing sepsis as the common pathway to death and disability from most infections is not just cosmetic packaging, but has relevance in its recognition and treatment. Regardless of its cause, the signs and symptoms of sepsis are nonspecific, following a pattern; treatment options are limited (e.g., antimicrobial agents, fluids, oxygen and cardiorespiratory support).

Many factors set sepsis apart from other conditions. No one medical discipline is tasked with addressing sepsis, but patients in all areas are vulnerable. Sepsis can be prevented by simple means, such as hand hygiene, perinatal care, clean deliveries, attention to nutrition (e.g., exclusive breastfeeding in infants), vaccines and appropriate health-seeking behaviours. Most importantly, for best outcomes, treatment of sepsis is time sensitive and should be started before laboratory data confirm the diagnosis. ${ }^{1}$ The approach to diagnosis and treatment (simple bundled therapy) can be taught easily to health care workers and offered in both resource-rich and -poor environments. ${ }^{4}$

About $25 \%-30 \%$ of people will die during an acute episode of sepsis, with higher rates in low-income countries. ${ }^{1}$ Major determi- nants of death include late diagnoses, poor health-seeking behaviours and lack of access to health care. Survivors of an acute episode are more likely to be readmitted or die in the early postdischarge period, and often go on to have pain, neurologic impairment, posttraumatic stress or functional disability.

Sepsis is not simply a clinical disease; it is also a social, an economic and a political disease, as indicated by a recent United Nations resolution. ${ }^{4}$ The situation in Canada is no different from any other part of the world where inequity in access and quality of care exist. In our large cities, tertiary and quaternary care facilities follow established guidelines and protocols; however, Canada's large land mass and remote communities, in which health care may be suboptimal, render sepsis a critical problem for many Canadians.

By raising awareness among the public, policy-makers and clinicians, by sharing ideas, and by bringing together like-minded people who are passionate about fixing this problem, CMAJ intends to do its part to highlight sepsis and improve clinical outcomes for those affected in Canada and worldwide.

Focusing on these four areas does not mean CMAJ is changing its editorial direction. Indeed, CMAJ has regularly published on these topics during its long history. Nor is CMAJ uninterested in other areas of medicine or health care. As always, we welcome submissions on topics of broad interest to our large readership.

Over the next months, CMAJ will launch initiatives on mental health, health services and the health of vulnerable populations. We need your ideas, suggestions, energy and submissions as we move forward. For more on what CMAJ is doing in our areas of focus and how you can contribute, see cmaj.ca.

\section{References}

1. Dugani S, Veillard J, Kissoon N. Reducing the global burden of sepsis. CMAJ 2017;189:E2-3.

2. Fotheringham JT. Primary pyelitis in infants. Can Med Assoc J 1911;1:1155-61.

3. Singer M, Deutschman CS, Seymour CW, et al. The Third International Consensus Definitions for Sepsis and Septic Shock (Sepsis-3). JAMA 2016;315:801-10.

4. Reinhart K, Daniels R, Kissoon N, et al. Recognizing sepsis as a global health priority - A WHO resolution. N Engl J Med 2017;377:414-7.

Competing interests: See www.cmaj.ca/site/misc/cmaj_staff.xhtml. Niranjan Kissoon is Vice-Chair of the Global Alliance for Sepsis.

Affiliations: Editor-in-Chief [interim] (Kelsall), CMAJ; University of British Columbia and British Columbia Children's Hospital (Kissoon), Vancouver, BC

Correspondence to: CMAJ editor, pubs@cmaj.ca 